\title{
Probing explosion geometry of core-collapse supernovae with light curves of the shock breakout
}

\author{
Akihiro Suzuki $^{1}$ and Toshikazu Shigeyama ${ }^{2}$ \\ ${ }^{1}$ Center for Computational Astrophysics, National Astronomical Observatory of Japan, Japan \\ email: asuzuki@cfca.jp \\ ${ }^{2}$ Research Center for the Early Universe, School of Science, University of Tokyo, Japan
}

\begin{abstract}
We consider supernova shock breakout in aspherical core-collapse supernovae. We perform hydrodynamical calculations to investigate the propagation of a strong shock wave in a compact star and the subsequent emergence from the surface. Using the results combined with a simple emission model based on blackbody radiation, we clarify how aspherical energy depositions affect shock breakout light curves.
\end{abstract}

Keywords. supernovae: general, shock waves, X-rays: bursts

\section{Introduction}

The final evolutionary state of a massive star is a violent explosion of the star as a core-collapse supernova. The gravitational collapse of the iron core triggered by photodisintegration process deposits the gravitational energy into the central region of the star, which generates a strong shock wave propagating in the stellar interior. The emergence of the shock wave from the surface is accompanied by a UV/X-ray flash, which is known as a supernova shock breakout.

We observe supernovae by using electromagnetic radiation only after the shock breakout phase, which means that detection of the shock breakout emission is very difficult. However, recent developments in observational techniques have gradually allowed us to detect such phenomena. On the theoretical side, there are lots of earlier studies on shock breakout emissions: pioneering works by Grassberg et al. (1971), Arnett \& Falk (1976), Chevalier (1976), Falk (1978), and Klein \& Chevalier (1978); semi-analytical considerations by e.g., Matzner \& McKee (1999); and one-dimensional radiation-hydrodynamical calculations by Shigeyama et al. (1988), Ensman \& Burrows (1992), and so on. Recently, several effects on temporal and spectral features of shock breakout emissions have been extensively considered. For example, the importance of the deviation of the shocked matter from thermal equilibrium, which is crucial for predicting spectra of shock breakout emission, has been investigated by, e.g., Katz et al. (2010), Nakar \& Sari (2010). The so-called bulk comptonization process may cause the deviation in the spectra from the Planck function (Wang et al. 2007; Suzuki \& Shigeyama 2010). Moriya et al. (2011) and Chevalier \& Irwin (2011) considered shock breakout in a dense wind. In addition, results from 1D radiation-hydrodynamical calculations have been compared in detail with the observed shock breakout emission from a type II supernova, SNLS-04D2dc (Tominaga et al. 2009).

However, most works have assumed spherical symmetry. In fact, deviation from spherical symmetry is also important in predicting shock breakout light curves. The importance is pointed out by Couch et al. (2009), Suzuki \& Shigeyama (2010), and Couch et al. 


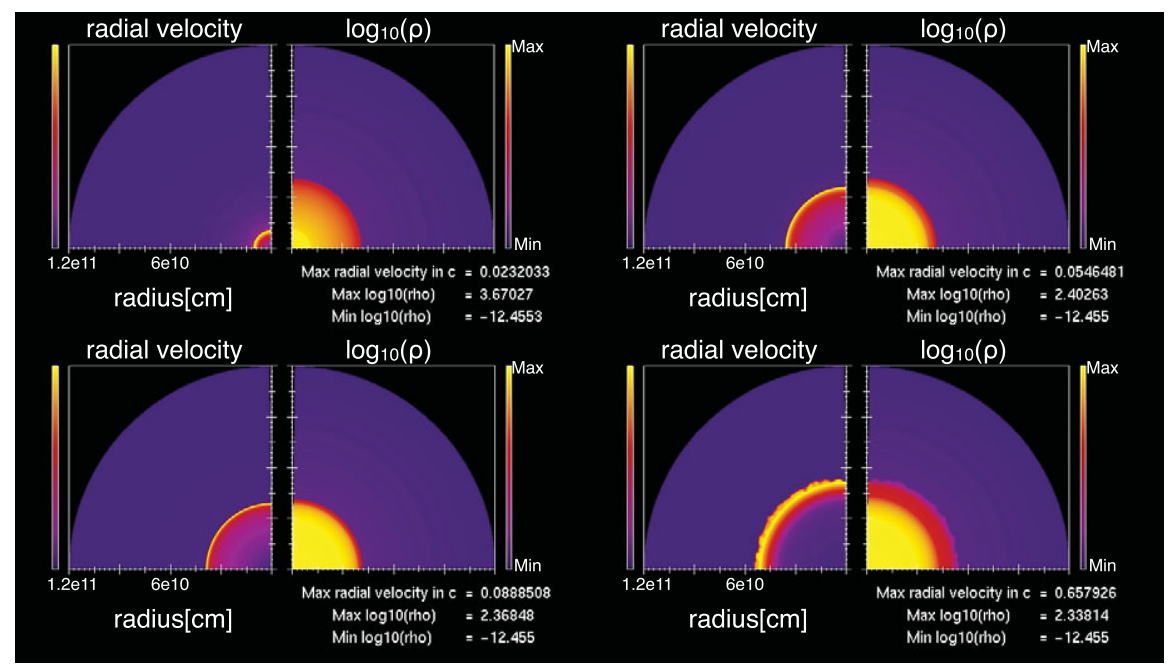

Figure 1. Snapshots of radial velocity and density maps at $t=1$ (top left), 36 (top right), 37 (bottom left), and 38 (bottom right) sec in the spherical case $(\alpha=0)$.

(2011). In this letter, we show results from hydrodynamical calculations of aspherical shock breakout on the surface of a compact progenitor, which is expected to explode as a type Ic supernova.

\section{Hydrodynamical calculations}

Hydrodynamical calculations are performed by using a two-dimensional special relativistic hydrodynamics code in spherical coordinates $(r, \theta)$ developed by one of the authors. The radial coordinate $r$ ranges from $r_{\text {in }}=3 \times 10^{8} \mathrm{~cm}$ to $r_{\text {out }}=1.2 \times 10^{11} \mathrm{~cm}$ and the angular coordinate $\theta$ from 0 to $\pi / 2$. The computational domain is covered by $1024 \times 128$ meshes. The progenitor model is a $14 \mathrm{M}_{\odot} \mathrm{CO}$ core with a radius of $4 \times 10^{10}$ $\mathrm{cm}$, which is taken from Woosley \& Heger (2006). For the equation of state, an ideal gas with an adiabatic index of $\gamma=4 / 3$ is assumed. We inject an energy of $E_{\text {total }}=10^{51}$ ergs from the inner boundary as the kinetic energy. Aspherical explosions are realized by assuming the following condition on the kinetic energy flux at the inner boundary,

$$
\frac{\rho_{\text {in }} v_{r}^{3}}{2}=\frac{E_{\text {total }}}{4 \pi r_{\text {in }}^{2} \tau_{\text {in }}}[1+\alpha \cos (2 \theta)] \text { for } t<\tau_{\text {in }} .
$$

Here $\rho_{\text {in }}$ is the density at the inner boundary and $\tau_{\text {in }}$ is the duration of the energy injection, which is fixed to be $0.1 \mathrm{sec}$ in this study. The introduced parameter $\alpha(0 \leqslant$ $\alpha \leqslant 1.0$ ) controls the asphericity of the explosion. For $\alpha>0$, a jet-like explosion is realized.

In this paper, we show results of our calculations with $\alpha=0$ and 0.8 (referred to as spherical and aspherical cases hereafter). Figure 1 represents snapshots of radial velocity and density maps in the spherical case. In this case, the shock breakout occurs at all points on the stellar surface in the same manner. In Figure 2, snapshots at similar epochs in the aspherical case are shown. One can see clear deviations from the spherical case arising from the aspherical energy deposition at the core. 


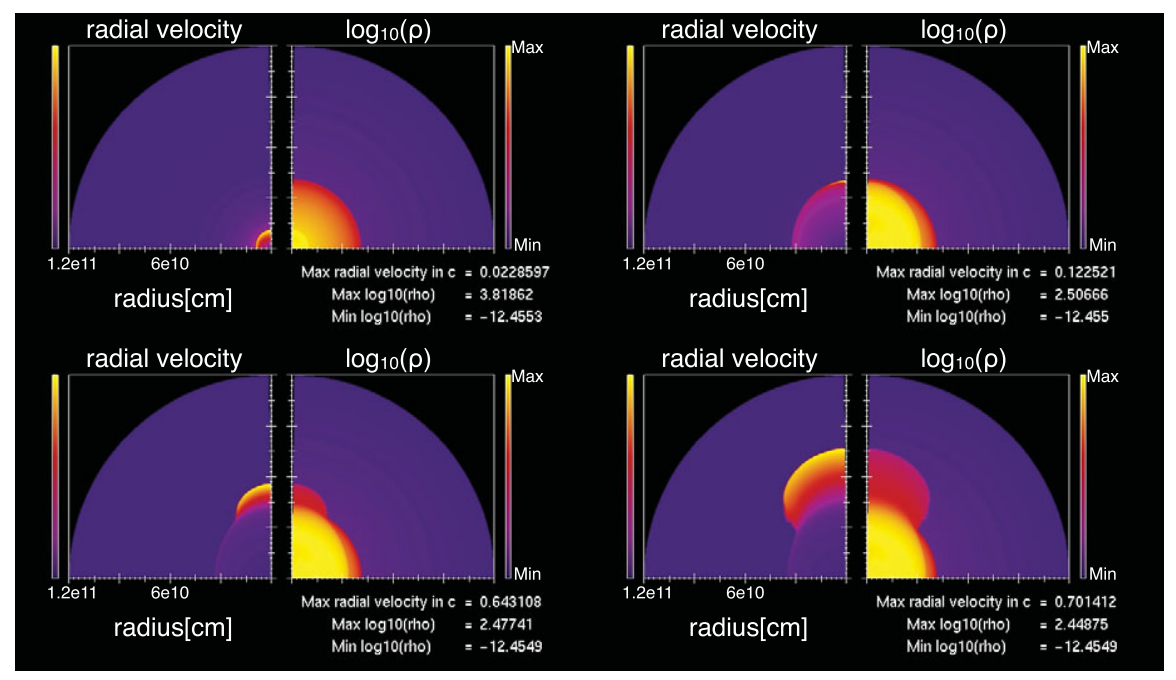

Figure 2. Snapshots of radial velocity and density maps at $t=1$ (top left), 35 (top right), 36 (bottom left), and 37 (bottom right) sec in the aspherical case $(\alpha=0.8)$.

\section{Calculation of light curves}

We calculate light curves of the shock breakout emission as seen by distant observers in the following simple way. We consider two observers located 100 stellar radii away from the star with inclination angles $\Theta=0^{\circ}$, and $90^{\circ}$. At first, results of our 2D calculations are mapped into 3D space. Next, we identify the photosphere, where the Thomson optical depth measured from each observer is unity. Assuming that black body radiation is emitted from the photospheres, we calculate light curves of the emission. Here, the radiation temperature $T_{\mathrm{ph}}$ is derived by assuming that the pressure $p$ at the photosphere is dominated by the radiation pressure, $p=a_{\mathrm{r}} T_{\mathrm{ph}}^{4} / 3$, where $a_{\mathrm{r}}$ is the radiation constant.

Figure 3 represents the resultant light curves. As shown in the left panel of the figure, in the case with the viewing angle of $\Theta=0^{\circ}$, the shock breakout emission is less luminous than the spherical case and the luminosity rapidly decreases at $t \sim 173 \mathrm{~s}$. On the other hand, in the case with the viewing angle of $\Theta=90^{\circ}$ (the right panel), the light curve shows a plateau-like feature after the rising and then the luminosity gradually approaches that of the spherical case.

\section{Discussions and conclusions}

In the previous sections, we have seen that the aspherical energy deposition at the core of a compact star affects its shock breakout emission. Then, the question is what makes the differences between the light curves. In fact, the differences arise from a simple geometrical effect as explained in the following.

At first, we consider the aspherical case with the viewing angle of $\Theta=0^{\circ}$. As seen in Figure 2, the shock breakout occurs in the region around the jet axis at first. In this case, the area of the emitting region is smaller than that in the spherical case, where the shock breakout simultaneously occurs at all points on the surface. This makes the luminosity lower than that in the spherical case. After the emergence of the shock wave from the surface, the ejected matter rapidly expands and then covers the star, which prevents the observer from seeing the shock breakout emission from the region near the equatorial plane. Therefore, the observer sees the emission from the cooling ejecta instead of the 

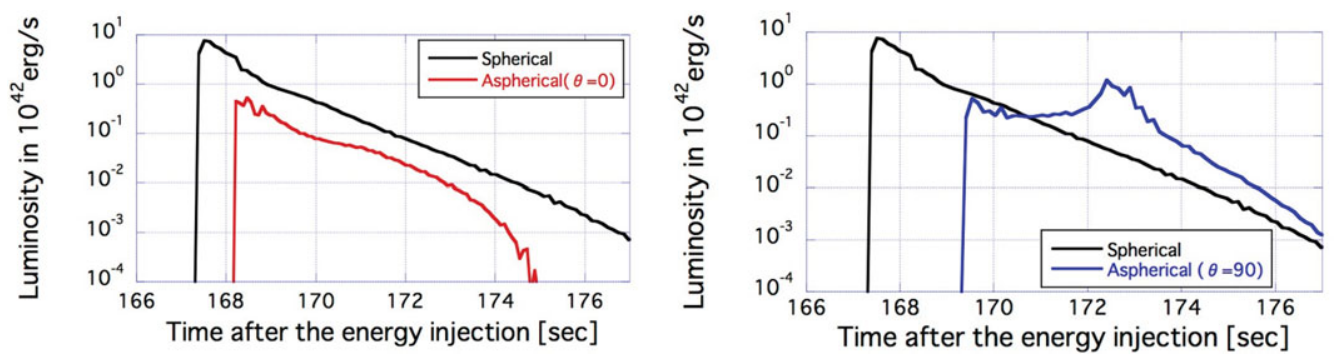

Figure 3. Luminosities of the shock breakout emissions as a function of time measured from the energy injection. In the left panel, light curves of the spherical case and the aspherical case with a viewing angle $\Theta=0^{\circ}$ are compared. In the right panel, same as the left panel but with a viewing angle $\Theta=90^{\circ}$.

shock breakout emission. This is the reason why the luminosity drops at $t \sim 173 \mathrm{~s}$ as seen in the left panel of Figure 3.

On the other hand, in the case of the viewing angle of $\Theta=90^{\circ}$, the observer can see the shock breakout occuring near the equatorial plane. Furthermore, the duration of the shock breakout emission becomes longer than that of the spherical case because the emergence of the shock wave from the surface near the equatorial plane is delayed from that around the jet axis. This delay corresponds to the duration of the plateau-like phase seen in the light curve of the right panel of Figure 3.

Finally, we address the caveat of how the calculations of the light curves are made. As pointed out by several authors, deviation from thermal equilibrium may be important in the shocked envelope. In other words, the shocked matter may outshine in a way different from the black body radiation assumed here. Thus, we should note that predicting spectra of shock breakout emission in the simplified manner as was done in this study has large uncertainties. However, the behavior of light curves revealed here should be seen even when we include such effects, because they merely arise from geometrical effects.

\section{References}

Arnett, W. D. \& Falk, S. W. 1976, ApJ, 210, 733

Chevalier, R. A. 1976, ApJ, 207, 872

Chevalier, R. A. \& Irwin, C. M. 2011, ApJL, 729, L6

Couch, S. M., Wheeler, J. C., \& Milosavljević, M. 2009, ApJ, 696, 953

Couch, S. M., Pooley, D., Wheeler, J. C., \& Milosavljević, M. 2011, ApJ, 727, 104

Ensman, L. \& Burrows, A. 1992, ApJ, 393, 742

Falk, S. W. 1978, ApJL, 225, L133

Grassberg, E. K., Imshennik, V. S., \& Nadyozhin, D. K. 1971, ApSS, 10, 28

Katz, B., Budnik, R., \& Waxman, E. 2010, ApJ, 716, 781

Klein, R. I. \& Chevalier, R. A. 1978, ApJL, 223, L109

Matzner, C. D. \& McKee, C. F. 1999, ApJ, 510, 379

Moriya, T., Tominaga, N., Blinnikov, S. I., Baklanov, P. V., \& Sorokina, E. I. 2011, MNRAS, 415,199

Nakar, E. \& Sari, R. 2010, ApJ, 725, 904

Shigeyama, T., Nomoto, K., \& Hashimoto, M. 1988, A\&SAp, 196, 141

Suzuki, A. \& Shigeyama, T. 2010, ApJL, 717, L154

Suzuki, A. \& Shigeyama, T. 2010, ApJ, 719, 881

Tominaga, N., Blinnikov, S., Baklanov, P., et al. 2009, ApJL, 705, L10

Wang, X.-Y., Li, Z., Waxman, E., \& Mészáros, P. 2007, ApJ, 664, 1026

Woosley, S. E. \& Heger, A. 2006, ApJ, 637, 914 